\title{
Internacionalização do ensino superior e os impactos da imigração na saúde mental de estudantes internacionais
}

\author{
Internationalization of higher education and the impact \\ of immigration on mental health of international students
}

\begin{abstract}
Alisson Vinícius Silva-Ferreira ${ }^{1}$
${ }^{1}$ Universidade Federal de Santa Catarina | Programa de Pós-Graduação em Psicologia

Foz do Iguaçu | PR | Brasil. Contato: alisson.psferreira@gmail.com

https://orcid.org/0000-0003-0634-6072
\end{abstract}

Lucienne Martins-Borges ${ }^{2}$

${ }^{2}$ Université Laval | École de travail social et de criminologie

Quebec | Canadá. Contato: lucienne.martins-borges@tsc.ulaval.ca

http://orcid.org/0000-0003-4323-116X

\author{
Thiago Guedes Willecke ${ }^{3}$ \\ ${ }^{3}$ Universidade Federal de Santa Catarina | Faculdade de Filosofia e Ciências Humanas | \\ Departamento de Psicologia \\ Florianópolis | SC | Brasil. Contato: thiagogwillecke@gmail.com \\ http://orcid.org/0000-0002-9952-3850
}

Resumo: No cenário educacional contemporâneo está cada vez mais presente o fluxo de estudantes que almejam ampliar seus horizontes educacionais cursando o ensino superior em outro país. Com a imigração e a mudança de país e cultura, o estudante pode estar sujeito a um estado de vulnerabilidade psíquica devido à perda dos referenciais culturais. Nesse sentido, e decorrente do fenômeno de internacionalização do ensino superior, o presente estudo teve como objetivo investigar quais são os impactos da imigração na saúde mental de estudantes internacionais e quais os fatores de risco e de proteção à saúde mental destes estudantes. Para tal, foi realizada revisão de literatura em três bases de dados com estudos nacionais e internacionais, sendo elas: BVS-Psi, Redalyc e EBSCO. Os resultados apontaram uma diversidade de fatores individuais, sociais, institucionais e culturais que impactam na saúde mental dos estudantes internacionais, cujas expressões de sofrimento psíquico se concentraram em estresse decorrente da adaptação ao novo contexto cultural e acadêmico, acompanhado por mal-estar psíquico, sintomas somáticos, dificuldades cognitivas e quadros clínicos de depressão, ansiedade e estresse pós-traumático. Observou-se ainda a subutilização dos serviços de apoio psicológico nas universidades, a demanda no início do curso, a diferença cultural na expressão dos sintomas e a maior procura por ajuda entre estudantes mulheres, em relação a homens. Em conclusão, o estudo aponta a necessidade de programas de acolhimento e integração dos estudantes internacionais que possibilitem minimizar o impacto migratório inicial e assim colaborar para a adaptação socioeducacional do estudante.

Palavras-chave: Imigrantes. Mobilidade acadêmica. Saúde mental do estudante.

Abstract: In the contemporary educational scenario, the flow of students seeking to broaden their horizons in higher education in another country is increasingly present. With immigration and the change of country and culture, the student may be subject to a state of psychic vulnerability due to the loss of cultural references. In this sense, and due to the phenomenon of internationalization of higher education, the present study aimed to investigate what are the impacts of immigration on the mental health of international students and what are the risk factors and protection of mental health of these students. For this purpose, a literature review was carried out in three databases with national and international studies: BVS-Psi, Redalyc and EBSCO. The results pointed to a diversity of individual, social, institutional and cultural factors that impact on the mental health of international students, whose expressions of psychic suffering focused on the stress of adaptation to the new cultural and academic context, accompanied by psychic malaise, somatic disorders, cognitive difficulties, and clinical manifestations of depression, anxiety, and post-traumatic stress. It was also verified the underutilization 
of psychological support services in universities, the demand at the beginning of the course, the cultural difference in the expression of symptoms and the greater demand for help among female students. In conclusion, the study points out the need for programs to host and integrate international students that make it possible to minimize the initial migratory impact and thus to collaborate for the socioeducational adaptation of the student.

Key words: Immigrants. Academic mobility. Student mental health.

DOI: http://dx.doi.org/10.1590/S1414-40772019000300003

Este é um artigo publicado em acesso aberto sob uma licença Creative Commons https://creativecommons.org/licenses/by-nc/4.0/

Recebido em: 23 de janeiro de 2019

Aprovado em: 1 de outubro de 2019

\section{Introdução}

No surgimento da universidade no ocidente, no século XI em Bolonha, esta caracterizou-se pela presença de professores e estudantes de várias partes do mundo, favorecendo a construção e socialização do conhecimento para além das fronteiras geográficas. Com o surgimento da globalização, os imperativos econômicos acabaram por exigir cada vez mais uma educação voltada para uma "aldeia global", conectada e com intensas trocas comerciais e culturais (ZAMBERLAM et al., 2009).

No Brasil, segundo dados do instituto nacional de estudos e pesquisas educacionais (INEP, 2017), em 2015 havia 15.605 estudantes internacionais no ensino de graduação, oriundos de 174 nacionalidades diferentes, dos quais a maioria (32\%) era do continente americano. Também segundo o INEP (2017), as duas instituições de ensino superior do Brasil com maior número de estudantes internacionais em 2015 eram a Universidade Federal da Integração Latino-Americana (UNILA) e a Universidade Federal da Integração Internacional da Lusofonia Afro-Brasileira (UNILAB). Ambas foram criadas em 2010, com um paradigma de vocação internacional e integração regional entre os países do hemisfério sul, atendendo aos pressupostos de uma educação voltada para a alteridade e a internacionalização solidária.

No Brasil, outros programas de internacionalização do ensino superior se fazem presentes, como o Programa de Estudantes-Convênio de Graduação (PEC-G), que oferece oportunidades de ensino superior a cidadãos de países em desenvolvimento com os quais o Brasil mantém acordos educacionais e culturais (GIRARDI; MARTINS-BORGES, 2017). Além disso, há também os programas de vagas para refugiados e/ou portadores de visto humanitário que algumas universidades federais oferecem; entre elas: Universidade Federal do ABC, Universidade Federal do Paraná, Universidade Federal de Santa Maria e a UNILA. 
Todavia, de forma intrínseca à questão da internacionalização do ensino superior está o fenômeno da imigração. Segundo definição da Organização Internacional de Migrações, o imigrante é aquele que, independente da causa e duração, atravessa a fronteira de outro país (OIM, 2009). Contudo, a respeito dos estudantes internacionais podemos caracterizá-los como aqueles que encontram na imigração a possibilidade de qualificação profissional e pessoal para a realização ou reconfiguração de um projeto de vida.

Destes estudantes, há uma parcela que planeja a entrada no ambiente universitário desde sua partida, ou seja, saem de seus países com o propósito de alcançar maiores possibilidades educacionais. Estas repercutem não apenas em um projeto que vislumbre maior qualidade de vida ao sujeito, mas podem estender seus efeitos à família e à própria comunidade de origem, visto que o retorno do universitário com os conhecimentos do país de imigração representa um impacto social em ambas. Porém, também há aqueles que após algum tempo residindo no país de destino, encontram na oportunidade do ensino superior a possibilidade de reconfiguração do seu projeto existencial.

Nesse sentido, conforme as características da imigração podemos ter no ambiente universitário: a) imigrantes que se movem de forma autônoma, ou seja, estudantes que utilizam aporte financeiro próprio para financiar seus estudos no exterior e fazem parte de uma elite econômica e educacional; b) estudantes que conseguem incentivos acadêmicos, que são aqueles que recebem bolsa de estudos e se deslocam dentro da lógica de cooperação educacional entre os países e instituições; c) os que migram por razões de vulnerabilidade econômica e reiniciam seu projeto de vida acadêmico; e por fim, d) os refugiados ou imigrantes humanitários, que foram violentamente forçados a deixarem seus países e que encontram no acesso à universidade uma oportunidade de recomeço (MARCHETTO 2003 apud ZAMBERLAM et al., 2009).

Entretanto, é relevante que ao se observar as motivações do imigrante para entrar em uma universidade estrangeira, sejam consideradas todas as variáveis de um processo de interação cultural mediado pela diferença, tal como língua, condições socioeconômicas, costumes, valores, sistemas educacionais diferentes, preconceito racial, étnico e de gênero. A imigração pode inaugurar um processo contínuo de estresse, decorrente a perda de elementos de proteção psíquica, como a cultura fundadora, a rede de apoio social, o status social anterior à imigração, a expressão em língua materna, entre outros (MARTINS-BORGES, 2013).

Desta forma, o imigrante está sujeito a mudanças subjetivas, ao não se reconhecer nos novos referenciais simbólicos. Para tentar diminuir este mal-estar, o imigrante inicialmente se esforça para compreender o mundo a sua volta, tentando se adaptar e incorporar os novos costumes e crenças, suprimindo muitas vezes sua própria bagagem cultural ou se isolando na 
convivência quase que exclusiva com pessoas da sua nacionalidade. Tal esforço para achar este lugar de pertencimento o predispõe a um aumento de sua vulnerabilidade, possibilitando emergir o sofrimento psíquico como expressão de sentido de tal condição de desamparo (GIRARDI; MARTINS-BORGES, 2017).

Diante destas considerações e pressupostos teóricos a respeito do processo de internacionalização do ensino superior e os impactos da imigração nos estudantes internacionais, que este estudo se dispôs a revisar a literatura nacional e internacional sobre os fatores de risco e proteção à saúde mental dos estudantes internacionais, bem como quais as principais expressões de sofrimento psíquico do impacto migratório. Assim, o estudo pretende colaborar com reflexões que auxiliem em ações no âmbito da atenção psicossocial ao estudante internacional e em programas e práticas de acolhimento institucional, local, nacional e/ou transnacional ao estudante internacional.

\section{Método e panorama do levantamento bibliográfico}

Com o objetivo de explorar o cenário de produção científica referente à saúde mental de estudantes internacionais, foi realizada a revisão de literatura em três bases de dados com sedes em diferentes localidades no continente americano. A primeira foi a Biblioteca Virtual em Saúde e Psicologia (BVS-Psi) que congrega estudos brasileiros a partir de bases como Lilacs, Index Psi, Scielo, Pepsic. Os descritores utilizados foram: Imigração e universitários; imigração e estudantes; saúde mental e imigrantes universitários; estudantes LatinoAmericanos; estudantes estrangeiros; estudantes imigrantes; imigrantes universitários; estudantes internacionais.

A segunda base de dados foi a Rede de Revistas Científicas da América Latina, Caribe, Espanha e Portugal (Redalyc), cujo os descritores foram: inmigración y universitários; inmigración y estudiantes; salud mental y estudiantes extranjeros; estudiantes Latinoamericanos; universitários inmigrantes; estudiantes inmigrantes; Estudiantes extranjeros; Estudiantes internacionales. E, por fim, a terceira base analisada foi a EBSCO, com sede nos Estados Unidos, tendo o Psychology and behavioral sciences collection como banco de dados, com os seguintes descritores: Immigration and university students; immigration and students; mental health and foreign students; Latin American students; immigrant students; international students and mental health.

O levantamento foi realizado entre julho de 2017 e fevereiro de 2018, e buscou artigos publicados entre os anos de 2007 e 2016. Decorrente os testes em cada base, foi considerado 
mais eficaz utilizar os descritores conforme a principal língua utilizada nas plataformas. Após seleção dos artigos pelos descritores, foi realizada a leitura de todos os títulos, resumos e palavras-chave daqueles que correspondiam aos seguintes critérios de inclusão: (1) ter relação com a temática de estudantes internacionais; (2) ser decorrente de pesquisa científica e avaliado por pares; (3) ter sido redigido em Português, Espanhol ou Inglês.

Foram encontrados, inicialmente, 256 artigos no BVS-Psi, porém após leitura completa, somente 11 atenderam os critérios acima. No Redalyc foram encontrados 55, dos quais 13 eram condizentes com os critérios de seleção, e 1167 na EBSCO, dos quais 67 atendiam aos critérios de inclusão, totalizando assim 91 artigos. Após esta análise e seleção, as pesquisas foram organizadas com o auxílio do software de gerenciamento de bibliografia Mendeley, onde foi possível verificar os artigos duplicados, restando assim 57. Esses foram submetidos à leitura integral, e mais dois artigos foram descartados por não atenderem ao critério de ter como objeto de estudo o fenômeno da imigração estudantil, resultando 55 artigos para a análise.

Referente aos participantes das pesquisas brasileiras, em 07 pesquisas estes eram imigrantes africanos, 03 envolviam nacionalidades de diversos continentes, 01 era com imigrantes asiáticos, e 01 exclusivamente com participantes dos demais países da América Latina. No total, as pesquisas foram produzidas nos seguintes países:

Tabela 1 - Países onde os estudos foram realizados

\begin{tabular}{cc}
\hline País & Número de Artigos \\
\hline EUA & 18 \\
Brasil & 11 \\
Reino Unido & 5 \\
Nova Zelândia & 3 \\
Canadá & 3 \\
Taiwan & 2 \\
Espanha & 2 \\
Alemanha & 1 \\
Austrália & 1 \\
Cuba & 1 \\
Argentina & 1 \\
Porto Rico & 1 \\
Paquistão & 1 \\
África do Sul & 1 \\
Japão & 1 \\
Hong Kong e Austrália & 1 \\
Austrália e Nova Zelândia & 1 \\
Jordânia e Israel & 1 \\
\hline
\end{tabular}


Dentre as pesquisas, 09 tinham participantes latino-americanos, sendo que a maioria das pesquisas (25) trabalhou com a população asiática. Além das pesquisas realizadas no território asiático, tal número é explicado, no caso dos EUA, pelo fato de que 46,3\% dos estudantes internacionais serem chineses, indianos ou sul coreanos (HWANG; BENNETT; BEAUCHEMIN, 2014).

Observa-se, que parece existir uma lacuna na literatura referente a produções científicas que compreendam o fenômeno da saúde mental em estudantes internacionais no Brasil; há também indicadores dessa lacuna nos outros países da América Latina e Caribe. Tal escassez pode ser compreendida decorrente do movimento histórico de emigração de estudantes da América Latina para países de maior investimento em ciência e tecnologia (Migração SulNorte), e de iniciativas apenas pontuais, descontinuadas e recentes que objetivem a troca de conhecimento entre os países do hemisfério sul (ZAMBERLAM et al., 2009). Segundo os autores, a "fuga de talentos" para os países industrializados e a reduzida mobilidade estudantil entre países latinos tem por consequência a baixa produção científica sobre a mobilidade dentro dos países da América Latina.

Por este ângulo, é possível conjecturar que, decorrente dos históricos entraves educacionais e tecnológicos em alguns países da América Latina e do Caribe, dos interesses do mercado de mão de obra qualificada e da valorização do conhecimento produzido em países industrializados, a internacionalização do ensino superior e as pesquisas desta área (no sentido do acolhimento) sejam ainda incipientes na literatura latino americana (ZAMBERLAM et al., 2009; PERROTTA, 2015).

Outro ponto a se considerar é que os fluxos de estudantes internacionais na globalização são influenciados por acordos econômicos e relações políticas entre os blocos e países. Fatores como a possibilidade de revalidação de diploma, o financiamento público (bolsas), a parceria entre universidades, a desburocratização no processo migratório, as demandas de mão de obra do país de recepção, as demandas por desenvolvimento científico dos países que enviam seus estudantes, o status econômico e tecnológico do país de acolhimento, a língua de aprendizagem e a regularidade no investimento em educação superior, influenciam diretamente no destino dos estudantes internacionais (ZAMBERLAM et al., 2009; PERROTTA, 2015).

Nesse sentido, a política de internacionalização no ensino superior pode ser atravessada por duas lógicas. A primeira segue os preceitos da Organização das Nações Unidas (ONU) para a Educação, Ciência e Cultura (UNESCO), que preconiza a educação como serviço público e, portanto, é destinada ao bem público e desenvolvimento social e igualitário da sociedade. Nesta diretriz ocorre a internacionalização solidária no ensino superior, que almeja a diminuição da 
pobreza por meio da educação e o desenvolvimento tecnológico e científico entre os países. Já a segunda lógica, preconizada pelo Banco Mundial e pela Organização Mundial do Comércio, compreende a educação enquanto mercadoria e, portanto, a internacionalização no ensino superior e o desenvolvimento da ciência e educação estariam subordinados aos interesses do mercado (ZAMBERLAM et al., 2009; PERROTTA, 2015). Assim, enquanto a internacionalização solidária seria guiada por princípios de inclusão social, por exemplo, para com os refugiados e estudantes oriundos de países com necessidades de desenvolvimento tecnológico e com a prerrogativa de retorno destes estudantes, a internacionalização segundo as lógicas do mercado estaria pautada na "captura de talentos" e na demanda e oferta de mão de obra, por exemplo (ZAMBERLAM et al., 2009; PERROTTA, 2015).

\section{Resultados e discussão}

Conforme leitura e análise das temáticas da literatura consultada, e objetivos da presente pesquisa, iremos apresentar uma síntese dos principais resultados das investigações científicas e suas contribuições para a compreensão dos impactos da imigração na saúde mental dos estudantes internacionais. Para tal, serão apresentados os potenciais fatores de risco à saúde mental deste grupo de imigrantes, assim como as expressões do sofrimento psíquico e os fatores de proteção.

A respeito dos fatores de proteção e de risco para a saúde mental, estes são variáveis presentes na relação de sujeitos e grupos com seu contexto, e influenciam no bem-estar. O conhecimento sobre tais variáveis é comumente utilizado em programas e intervenções para a promoção e prevenção em saúde, sendo que em intervenções com este fim, o paradigma de fortalecimento dos fatores de proteção vem se destacando como estratégia eficaz para a prevenção em saúde mental (ABREU; BARBALETTA; MURTA, 2015).

\section{Fatores de risco à saúde mental dos estudantes internacionais}

O primeiro fator destacado consistiu nas deficiências da preparação para a imigração. Um dos elementos que contribuiu para maior dificuldade de adaptação e uma intensificação do impacto migratório em estudantes internacionais foi a ausência de informações reais sobre onde pretendiam estudar e viver. A ausência de informações sobre o contexto institucional, cultural e social dos países e instituições de acolhida, e/ou a idealização destes contextos, se apresentaram, segundo as pesquisas que seguem, como intensificadoras do choque cultural destes estudantes (SOUZA; SEDIYAMA, 2012; OLIVENCIA, 2014) 
Em pesquisa realizada por Barbosa et al. (2016), sobre o acesso de estudantes internacionais ao Sistema Único de Saúde (SUS), os resultados indicaram que os estudantes desconheciam as formas de atendimento no SUS, o que gerava entradas por problemas menos graves nas Unidades de Pronto Atendimento (UPA), ao invés da busca por Unidades Básicas de Saúde. Tal problemática, segundo os autores, acabava por gerar uma incongruência entre as expectativas de tempo para o atendimento e resolutividade do problema.

Outro fator central que se relaciona com a preparação para a imigração diz respeito ao nível de proficiência da língua do país em que se irá estudar. Segundo Castañeda (2008), ao estudar em outra língua, o imigrante, a depender de seu domínio sobre esta, terá que dispor de mais tempo de estudo para compreender um texto ou conteúdo de sala de aula. Ao se expressar em uma língua que não domina, o estudante limita a expressão de seu pensamento, quando não prefere o silêncio ao ter que se expressar. A dificuldade linguística também se apresentou como um relevante fator na socialização e integração dos estudantes na comunidade acadêmica e na cidade que escolheram morar, o qual constituiu um fator para o isolamento social do imigrante em diversas pesquisas (CARRILLO, 2016; CHALUNGSOOTH; SCHNELLER, 2011; CHANG, 2007; DOKU; MEEKUMS, 2014; GRAYSON, 2008; PRIETO-WELCH, 2016; SILVA; MORAIS, 2012).

Ainda sobre o âmbito acadêmico, identificaram-se estressores enfrentados pelos estudantes internacionais decorrentes da necessidade de adaptação às diferentes metodologias de ensino e avaliação, do desconhecimento das regras e procedimentos acadêmicos, da escassez de documentos na língua materna, e da aprendizagem de conteúdos complexos em uma língua diferente (CASTAÑEDA, 2008; SANTIAGO et al., 2011). As diferenças acadêmicas, segundo Marginson (2014), muitas vezes podem ser agravadas por um etnocentrismo acadêmico, que consiste em uma intensa valorização dos elementos epistemológicos da instituição de acolhida e uma negação/indiferença aos conteúdos de formação educativa do estudante internacional. Tal posicionamento produz um verdadeiro "epistemicídio", que consiste no assassinato do conhecimento do outro (SANTOS, 2007).

No entanto, tal choque acadêmico, segundo Grayson (2008), também pode se relacionar com a qualidade da formação escolar do imigrante nos ensinos anteriores. Déficits na formação acadêmica potencializarão as dificuldades neste novo cenário educativo, os quais exigirão do estudante e da instituição um esforço maior para o desenvolvimento tanto do projeto acadêmico deste, quanto do processo de internacionalização da instituição.

Outro fator identificado para o aumento da tensão psicológica do estudante internacional se relaciona com a expectativa e pressão familiar e/ou comunitária por um sucesso acadêmico 
e retorno do estudante como "vitorioso". Nas investigações de Prieto-Welch (2016) e Subuhana (2009), destacou-se que estudantes internacionais tendem a buscar cursos de forte valorização social, e que a família tende a influenciar nesta decisão. No entanto, segundo os autores, à medida que o estudante internacional encontra dificuldade ao concretizar estas expectativas de sucesso depositadas pela família, este se depara com uma dívida simbólica e, em alguns casos, também com uma dívida real - financeira- para com os familiares.

Outro frequente resultado de pesquisa foi de que quanto maior a diferença cultural, maior a probabilidade de se encontrar dificuldades de adaptação/interação por parte dos estudantes internacionais (ALENCAR-RODRIGUES; STREY, 2010; HAN et al., 2013; OLIVENCIA, 2014; SUBUHANA, 2009; SWAMI, 2009). De acordo com os autores, a diferença cultural acaba, em um primeiro momento, por propiciar uma sensação de estranhamento da realidade externa e interna, e uma desestabilização afetivo-cognitiva no campo dos valores, hábitos e crenças. Segundo os estudos, esta desestabilização constitui um dos elementos centrais para a sensação de perda dos elementos culturais protetores. Em pesquisas realizadas por Alencar-Rodrigues e Strey (2010) e Barea (2008), a diferença nas concepções de gênero e sexualidade em cada país se configurou como elemento do impacto cultural na imigração. Neste caso, segundo o estudo de Barea (2008), a imigração de estudantes do sexo feminino (e pensando ainda a imigração de estudantes que diferem dos padrões heteronormativos, a depender de cada contexto), corresponderá a um movimento de rompimento com as amarras socioculturais dos países de origem e também de embate com a cultura do país de acolhimento.

Já a distância física da rede de apoio - família, amigos, companheiros - faz com que os estudantes internacionais estejam mais suscetíveis ao enfrentamento solitário dos problemas e dos estressores (DOKU; MEEKUMS, 2014; LU et al., 2015; PAN; WONG; YE, 2013). A ausência das paisagens, do clima e das comidas típicas também foi apresentada como fator do impacto cultural na pesquisa de Doku e Meekums (2014), propiciando, segundo os autores, sentimentos de desconexão e de não-pertencimento. A enfermidade de algum familiar ou pessoa significativa que ficou no país de origem, e a impossibilidade de retorno imediato também foram notificadas como estressores para os estudantes internacionais (OLIVENCIA, 2014).

Do mesmo modo, a dificuldade financeira surge como um agravante do estado de vulnerabilidade psicossocial de estudantes internacionais oriundos de classes sociais menos favorecidas economicamente (CARRILLO, 2016; SWAMI, 2009). Castañeda (2008), em seu estudo com estudantes argentinos nos EUA, observou que estes apresentavam maior dificuldade financeira que os autóctones. Dentre outros fatores, a valorização de cada moeda 
acaba por influenciar a relação entre a renda familiar ou bolsa do estudante e seu custo de vida no país de destino e, assim, suas possibilidades de ter acesso ao lazer, moradia e alimentação de qualidade.

Outro aspecto apontado nos estudos de Santiago et al. (2011), Hagelskamp, SuárezOrozco e Hughes (2010) e Saladino e Martinez (2015), diz respeito à possibilidade de trabalho formal ou não por parte do estudante internacional que necessitará trabalhar para conseguir se sustentar. Tal possibilidade estará atrelada ao tipo de visto obtido ao entrar no país. No caso do Brasil, o Conselho Nacional de Imigração, através da resolução normativa 124 do dia 13/12/2016, regulou a possibilidade de trabalho para estudantes com visto temporário. Nestas situações, é necessário, dentre outros critérios, que a função laboral tenha relação com o currículo acadêmico (BRASIL, 2018). Importante ressaltar que estudantes com visto humanitário ou status de refugiado já estão autorizados ao trabalho.

Uma ampla gama de pesquisas traz o preconceito racial como fator de risco para o sofrimento psíquico dos estudantes internacionais. Pesquisas brasileiras com estudantes africanos, realizadas por Maciel (2010), Mungoi (2012), Silva e Morais (2012), Subuhana, (2009) ratificaram que, apesar de sermos um país composto por fortes heranças africanas oriundas de uma violenta colonização, ainda vivenciamos o "mito da democracia racial" como apontado por Calligaris (1991), visto não termos superado o fantasma de um passado escravagista. Participantes relataram sentir maior preconceito fora do que dentro da universidade, e de forma mais explícita do que velada, além da intersecção entre preconceito racial e o de classe, pelo qual os estudantes africanos foram submetidos (SILVA; MORAES, 2012; SUBUHANA, 2009). Os estudantes relataram que o preconceito era atenuado quando eram reconhecidos como universitários e estrangeiros e não como "negros brasileiros"; ou seja, sentiam um preconceito menor ao saírem do estereótipo social de marginalização do negro no Brasil.

Todavia, não foram apenas as pesquisas brasileiras que notificaram em seus resultados a frequência da discriminação racial contra estudantes internacionais como fator de risco à saúde mental destes, e como campo constitutivo e interseccional da xenofobia. Conforme Carrillo (2016) e Prieto-Welch (2016), este também foi um fator encontrado em seus estudos. Já a discriminação étnica e religiosa também se apresentou como fator relacionado com o malestar dos estudantes internacionais, nas investigações de Nilsson, et al. (2008), Swami (2009), Hsien-Chuan et al. (2009). Sob o mesmo ponto de vista, conforme pesquisa de Carrillo (2016) com estudantes latino-americanos nos EUA, foi constatado o preconceito de classe como um dos principais desconfortos dos participantes em sua integração na sociedade estadunidense. 
Já na pesquisa de Woodward (2010), com estudantes refugiados de guerra na Inglaterra, foi apontado que os traumas anteriores não elaborados se apresentam como fatores de risco à saúde mental, visto o enfraquecimento das defesas psíquicas no processo migratório. Tal estudo indicou que mesmo diante da simbolização do curso universitário como desejo de desenvolvimento e de superação de sentimento de culpa e de sofrimento do passado, os estressores decorrentes da imigração e da instituição acadêmica podem remeter os estudantes a terem que elaborar traumas até então reprimidos.

Desse modo, segundo as pesquisas acima, podemos nomear que os principais fatores de risco na imigração estudantil são: deficiências na preparação linguística, diferença cultural, ausência de informações sobre o novo contexto, necessidade de adaptação a novas metodologias de ensino, etnocentrismo acadêmico, déficits na formação acadêmica, pressão familiar, diminuição da rede de apoio, dificuldade financeira, preconceitos e traumas anteriores. Conforme discutido, cada um destes fatores pode conter diversas especificidades.

\section{O impacto migratório e a saúde mental}

Destaca-se na literatura revisada que os impactos na saúde mental estão representados por expressões de sofrimento psíquico (emoções e sentimentos), por categorias diagnósticas, por demandas em serviços institucionais e, por fim, em uma única pesquisa, por efeitos positivos na saúde mental e na personalidade devido à imigração.

A pesquisa realizada por Swami (2009) com estudantes malasianos e chineses na GrãBretanha verificou que o nível de saúde se correlacionava com o nível da adaptação sociocultural no país anfitrião. Paralelamente, o estresse aculturativo provocado pela necessidade de adaptação ao novo ambiente cultural foi indicado como característica frequente para manifestações de sofrimento e sintomas nos estudantes internacionais (BAREA, 2008; LI et al., 2016; SHIM et al., 2014; SUN, 2013).

Já o sofrimento psíquico dos estudantes internacionais relatados nas pesquisas se concentraram em: sentimento de frustração, impotência, inadequação, isolamento, medo de fracassar, solidão, nostalgia, tristeza, perda, desânimo, desamparo, desesperança, ódio, sensação de não pertencimento, incerteza, estranhamento e desconexão (CARRILLO, 2016; CASTAÑEDA, 2008; DOKU; MEEKUMS, 2014; LI et al. 2016; OLIVENCIA, 2014; PRIETO-WELCH, 2016; SUBUHANA, 2009; SUN, 2013).

Quanto aos sintomas físicos, destacaram-se gastrite, cefaléia, virose, pico de hipertensão arterial, (BARBOSA et al., 2016) e também sintomas cognitivos, como fadiga cognitiva e 
dificuldade de concentração (PRIETO-WELCH, 2016). Outro ponto destacado nos estudos foi que a forma de expressão sintomática pode variar conforme a cultura de cada população. Nas pesquisas de Chang (2007) e Shim et al. (2014), verificou-se que os estudantes chineses apresentavam maior grau de manifestações somáticas do que cognitivo-afetivas, em comparação com outros estudantes internacionais. Nas mesmas pesquisas, somadas ao estudo de Hwang, Bennett e Beauchemin (2014) identificou-se que as mulheres procuram mais os serviços de aconselhamento psicológico do que os homens, e que o estigma em relação à saúde mental e às formas de tratamento em cada cultura influenciam a busca de apoio por parte dos estudantes internacionais.

Já o estudo de Andrade e Teixeira (2009), indicou que os estudantes internacionais estão mais propensos a buscar serviços de apoio na primeira metade do curso. Na mesma pesquisa, os principais serviços procurados foram: orientação e planejamento de carreira, assistência à busca de moradia, assistência médica/odontológica, espaço de convivência para estrangeiros, assistência psicológica e assistência jurídica.

Yakushko, Davidson e Sanford-Martens (2008), identificaram, em uma universidade estadunidense, que apenas $1,8 \%$ dos estudantes internacionais utilizavam o serviço de apoio psicológico da universidade, e que estes encerravam o tratamento mais precocemente do que os estudantes locais. Apontou-se também que, dentre os estudantes internacionais, os latinoamericanos e caribenhos tendiam a participar de um número maior de sessões. Todavia, a pesquisa de Chang (2007) com estudantes chineses em Taiwan também detectou uma subutilização dos serviços de saúde da universidade por parte dos estudantes internacionais, assim como na pesquisa de Lee e Pistole (2014) que notificou que, além da subutilização do serviço, a demora pela procura implicou em um agravo da saúde mental e do desempenho acadêmico dos estudantes internacionais.

Em termos de quadros sintomáticos, a depressão foi identificada em seis pesquisas (CHANG, 2007; HAN et al., 2013; PRIETO-WELCH, 2016; WOODWARD, 2010; YAKUSHKO; DAVIDSON; SANFORD-MARTENS (2008). Outros quadros clínicos relatados foram ansiedade, nas pesquisas de Sun (2013); Han et al. (2013) e estresse póstraumático com ideação suicida (WOODWARD, 2010).

Na pesquisa de Yakushko, Davidson e Sanford-Martens (2008), realizada em um centro de aconselhamento psicológico de uma universidade estadunidense, as principais demandas foram problemas de relacionamento $(22 \%)$ e depressão $(15,2 \%)$, seguidas de quadros de ansiedade, auto-estima, dificuldades acadêmicas e de orientação profissional, isolamento, estresse, imagem corporal e alimentação ( $4,6 \%$ a $6,9 \%$ cada); e por fim, demandas oriundas de 
traumas, preocupações com o retorno, abuso sexual, abuso de substâncias, identidade de gênero e ideações suicidas. Já na pesquisa de Hwang, Bennett e Beauchemin (2014) às principais demandas do centro de aconselhamento foram respectivamente: problemas de relacionamento (397), ansiedade (366), humor deprimido (346), problemas de adaptação em geral (313), problema com o controle dos impulsos (160) e de autoimagem (105).

Todavia, é importante citar, conforme pesquisa realizada por Pan, Wong e Ye (2013), com estudantes chineses pós-migração, que a imigração também possibilita crescimento intrapessoal e interpessoal. Segundo os autores, o processo migratório possibilitou aos participantes que desenvolvessem maiores recursos para superação de situações estressantes, metas de vida mais claras, autoconhecimento e autoconfiança. Foram também identificados o desenvolvimento de habilidades para a comunicação intercultural, aumento da rede social, apreciação do relacionamento familiar e maior compreensão das diferenças culturais.

Nesse sentido, as condições relacionadas ao sofrimento psíquico dos estudantes internacionais se concentraram no estresse decorrente da adaptação ao novo contexto cultural e acadêmico, acompanhado por mal-estar psíquico, sintomas somáticos, dificuldades cognitivas, quadros clínicos de depressão, ansiedade e estresse pós-traumático. Em relação às demandas nos serviços de aconselhamento psicológico destacaram-se as diferenças culturais na expressão dos sintomas, a subutilização dos serviços de apoio psicológico, a demanda no início do curso, e a diferença entre gêneros na busca por ajuda.

\section{Fatores de proteção à saúde mental dos estudantes internacionais}

Um dos fatores de proteção que se destacou nos estudos analisados foi a relação entre o estudante internacional e sua cultura no país de acolhimento. A possibilidade de expressar e preservar a herança cultural e a identidade através de vestimentas, da música, da dança, da comida, da expressão em língua materna, das festas típicas, reunião entre os pares, por exemplo, foram apresentados como proeminentes fatores para o amortecimento do sentimento de perda dos elementos identitários e da relação do sujeito com a realidade (DOKU; MEEKUMS, 2014; MACIEL, 2010; MUNGOI, 2012; SUBUHANA, 2009).

Do mesmo modo, os coletivos, as associações que recebem os estudantes internacionais e as redes virtuais construídas por estes para a recepção e integração dos recém-chegados, repercutem na redução do impacto cultural negativo e na construção de uma nova rede de apoio (SUBUHANA, 2009). No entanto, segundo Garcia (2012), apesar das amizades e do contato com pessoas do país de origem exercerem a importante função de diminuir o estresse decorrente 
o encontro intercultural via o compartilhamento dos mesmos elementos culturais, se a rede de apoio permanecer constituída apenas por pares, o estudante imigrante terá menos contato com a língua e a cultura anfitriã. Assim, a amizade, ou seja, o laço social dos estudantes internacionais com os estudantes nacionais se destacou como fator preponderante para a integração social nas investigações de Garcia (2012) e Swami (2009).

Da mesma forma, Kayitesi e Mwaba (2014), Maciel (2010) e Swami (2009) apontam que a abertura para a cultura local e o acesso aos espaços da cidade que permitam este aprendizado são fatores de extrema relevância para a possibilidade de integração cultural. Entretanto, segundo as investigações, a integração cultural também depende de uma cultura de hospitalidade por parte dos estudantes locais e dos autóctones em geral.

Sob a mesma perspectiva, atividades de lazer e esporte envolvendo estudantes internacionais e locais foram apontadas como instrumentos de integração, de favorecimento de laços de amizade e de contribuição para a diminuição dos sintomas de ansiedade e depressão em estudantes internacionais (SILVA; MORAIS, 2012; GARCIA, 2012). Ou seja, as atividades coletivas que possibilitam processos de identificação, sensações de pertencimento, satisfação e, ainda, os benefícios biológicos do esporte contribuem para a promoção da saúde de estudantes internacionais. Nota-se que em um primeiro momento, estes tendem a desconhecer as possibilidades de lazer e esporte dentro do novo território e estão em processo de construção de uma rotina, de novas amizades e, consequentemente, de inserção na sociedade local.

A religião/espiritualidade também demonstrou ser um recurso relevante para atenuar o desconforto psíquico. Alguns autores indicam que a prática religiosa se apresentou de forma mais acentuada nos estudantes internacionais do que nos estudantes nacionais, e que a religião exerce uma função de amparo psíquico e de atenuação dos estressores provocados pela nova realidade (HSIEN-CHUAN et al., 2009; PEI et al., 2012). A própria etimologia da palavra religião, que deriva do latim religare, se remete ao um movimento de "ligar novamente", ou seja, reconstituir uma aliança após uma separação, como uma forma de "ponte" entre o mundo interno e o mundo externo, entre o homem e os elementos considerados protetores e sagrados de sua cultura.

Os traços de personalidade também se apresentaram como fatores protetivos à saúde mental dos estudantes internacionais nas pesquisas de Marginson (2014) e Sun (2013). Características como tolerância à frustração, comunicabilidade, habilidades interpessoais, autoconceito positivo, introversão e extroversão, indicaram auxiliar os estudantes nas estratégias de adaptação e interação sociocultural. 
Já o acolhimento institucional, ou seja, as políticas de acolhimento e integração que a própria universidade desenvolve para a melhor inclusão dos estudantes internacionais, se apresentaram como um elemento de proteção psíquica. Os estudos apontaram para a importância de se desenvolver materiais sobre saúde mental traduzidos linguística e culturalmente, e também a valorização de práticas culturais dos imigrantes (CHALUNGSOOTH; SCHNELLER, 2011; LIMONTA VIDAL et al., 2012). Destacou-se igualmente a relevância do desenvolvimento de sensibilidade cultural nos técnicos administrativos (pedagogos, assistentes sociais, secretários acadêmicos, psicólogos, etc), professores e estudantes locais para o acolhimento cultural, linguístico e orientação, inclusive para possíveis encaminhamentos precoces para os serviços de apoio estudantil da universidade (ARAR, MASRY-HARZALLA; HAJ-YEHIA, 2012; SALADINO; MARTINEZ, 2015).

Neste mesmo sentido, segundo Yakushko, Davidson e Sanford-Martens (2008), o acolhimento docente, ou seja, a forma como o professor acolhe o estudante imigrante, se destaca como fator que repercute diretamente no sentimento de pertencimento, na confiança em se expressar em sala de aula e na busca de ajuda e informações. Este mesmo estudo também apontou para o trabalho de treinamento de professores-tutores como suporte aos estudantes durante o processo de adaptação. Assim, se destaca a importância do desenvolvimento de uma pedagogia que englobe e valorize a alteridade no processo formativo intercultural:

\footnotetext{
O futuro da educação, de fato, vai realizar-se exatamente nesse terreno, isto é, na capacidade de desconstruir o medo do Outro, colocando-se, contemporaneamente as bases para uma educação baseada no acolhimento, no respeito, no diálogo, na convivência, na procura/projetação de itinerários comuns tendentes à interdependência e ao enriquecimento recíproco. Em outros termos, somente programando, juntos, o caminho a fazer, será possível coeducar- "Nos" para a alteridade (PIERONI; FERMINO; CALIMAM, 2014, p. 215).
}

Sendo assim, os fatores de proteção à saúde mental dos estudantes internacionais concentraram-se na importância do contato com a própria cultura e abertura à nova cultura, o acolhimento por grupos de estudantes, a preparação da instituição e de seus serviços de apoio, a hospitalidade dos moradores da cidade, as amizades, o lazer, o esporte, a religião/espiritualidade, e, por fim, os traços de personalidade. Diante do exposto, e diante das diferentes perspectivas em que as pesquisas abordaram o fenômeno da imigração estudantil, ratificou-se que o processo migratório, mesmo em uma população aparentemente privilegiada, pode se tornar um fator de risco à saúde mental, a depender de um conjunto complexo e heterogêneo de fatores sociais, culturais, institucionais, políticos, geracionais e subjetivos. 


\section{Considerações finais}

Diante da análise da literatura consultada, constatou-se um reduzido número de pesquisas que investigaram a temática da saúde mental dos estudantes internacionais no Brasil. Porém, conforme os estudos nacionais e internacionais, ratificou-se a ocorrência de um impacto na saúde mental destes estudantes provocado pela imigração e a necessidade de adaptação e interação na nova cultura que, além de provocar sofrimento psíquico, tende a impactar no desempenho e desenvolvimento acadêmico destes estudantes.

Dessa maneira, cabe às instituições de ensino superior, dentro dos seus programas de internacionalização, atentar aos fatores de risco e proteção à saúde mental dos estudantes internacionais e, assim, desenvolver ações e projetos de acolhimento que possibilitem minimizar o impacto migratório e favorecer a integração e adaptação do estudante estrangeiro ao novo sistema de ensino-aprendizagem e ao novo ambiente sociocultural.

Dentre as potenciais ações a serem desenvolvidas pelas universidades, podemos destacar as estratégias de acolhimento e mediação linguístico-culturais a serem realizadas na chegada do imigrante na cidade e/ou na universidade (via os programas de apadrinhamento intercultural), a tradução de documentos e cartilhas de orientação, a formação para o desenvolvimento da competência cultural dos professores e funcionários, e também a manutenção da cultura via troca cultural com a comunidade mediante projetos de extensão e pesquisa que favoreçam o empoderamento do imigrante e o laço simbólico entre sua herança cultural e a sociedade local.

\section{Referências}

ABREU, Samia; BARLETTA, Janaína Bianca; MURTA, Sheila Giardini. Prevenção e promoção em saúde mental: pressupostos teóricos e marcos conceituais. In: MURTA, Sheila Giardini; LEANDRO-FRANÇA, Cristineide, SANTOS, Karine Brito dos; POLEJACK, Larissa. Prevenção e promoção em saúde mental: fundamentos, planejamento e estratégias de Intervenção. Novo Hamburgo: Sinopsys, 2015. v. 24.

ALENCAR-RODRIGUES, Roberta de; STREY, Marlene. Neves. Orquestrando vozes de gênero de estudantes estrangeiros/as latino-americanos/as. Psico, Porto Alegre, v. 41, n. 1, p. 47-56, jan./mar, 2010.

ANDRADE, Ana Maria Jung de; TEIXEIRA, Marco Antônio Pereira. Adaptação à universidade de estudantes internacionais: Um estudo com alunos de um programa de convênio. Rev. Bras. Orientac. Prof, Porto Alegre, v. 10, n. 1, p. 33-44, 2009. Disponível em: http://pepsic.bvsalud.org/scielo.php?script=sci arttext\&pid=S1679-33902009000100006. Acesso em: 21 jan. 2018. 
ARAR, Khalid; MASRY-HARZALLA, Asmahan; HAJ-YEHIA, Kussai. Higher education for Palestinian Muslim female students in Israel and Jordan: migration and identity formation. Cambridge Journal of Education, Cambridge, v. 43, n. 1, p. 51-67, 2012. Disponível em: tandfonline.com/doi/abs/10.1080/0305764X.2012.749391. Acesso em: 2 fev. 2018.

BARBOSA, Maiara Lima et al. Health care provided by SUS: the experience of foreign students. Escola Anna Nery - Revista de Enfermagem, Rio de Janeiro, v. 20, n. 4, 2016. Disponível em: http://www.scielo.br/scielo.php?pid=S141481452016000400210\&script=sci_arttext\&tlng=en. Acesso em: 20 jan. 2018.

BAREA, Eva Maria Gonzales. Un proceso migratorio estudiantil: jóvenes marroquies en la Universidad de Granada. Revista Electronica de Investigacion Educativa, Baja California, v. 10, n. 2, p. 1-13, 2008.

BRASIL. Resolução normativa CNIg 124/2016. Dispõe sobre a condição migratória temporária de estudante para a condição migratória temporária de trabalho. 2018. Disponível em: http://csbbrasil.org.br/downloads/CNIg/Resolucao-Normativa-n-124-DOU.pdf. Acesso em: 10 out. 2018.

CALLIGARIS, Contardo. Hello Brasil: notas de um psicanalista europeu viajando ao Brasil. São Paulo: Escuta, 1991.

CARRILLO, Juan F. Searching for "Home" in Dixie: Identity and Education in the New Latin@ South. Educational Studies, London, v. 52, n. 1, p. 20-37, 2016. Disponível em: https://www.tandfonline.com/doi/full/10.1080/00131946.2015.1120208?scroll=top\&needAcc ess=true. Acesso em: 21 fev. 2018.

CASTAÑEDA, Rosario Hernández. La experiencia de estudiar un posgrado en el extranjero (un estudio de caso). Revista Electrónica de Investigación Educativa, Baja California, v. 10, n. 2, p. 1-16, 2008.

CHALUNGSOOTH, Pornthip; SCHNELLER, Gregory. R. Development of Translation Materials to Assess International Students' Mental Health Concerns. Journal of Multicultural Counseling and Development, Virgínia, n. 39, p. 180-191, 2011. Disponível em: https://onlinelibrary.wiley.com/doi/abs/10.1002/j.2161-1912.2011.tb00150.x. Acesso em 31 jan. 2018.

CHANG, Hsaiowen. Depressive symptom manifestation and help-seeking among Chinese college students in Taiwan. International Journal of Psychology, London, v. 42, n. 3, p. 200-206, 2007. Disponível em: https://onlinelibrary.wiley.com/doi/full/10.1080/00207590600878665. Acesso em: 1 fev. 2018.

DOKU, Florence, MEEKUMS, Bonnie. Emotional connectedness to home for Ghanaian students in the UK. British Journal of Guidance and Counselling, London, v. 42, n. 4, p. 383-402, 2014. Disponível em: https://www.tandfonline.com/doi/abs/10.1080/03069885.2014.916396. DOI: https://doi.org/10.1080/03069885.2014.916396. Acesso em: 1 fev. 2018.

GARCIA, Agnaldo. Amizades de universitários estrangeiros no Brasil: um estudo exploratório. Estudos de Psicologia, Campinas, v. 29, n. 4, p. 471-479, out./dez. 2012. 
GIRARDI, Julia Freitas; MARTINS-BORGES, Lucienne. Dimensões do sofrimento psíquico em estudantes universitários estrangeiros. Psico, Porto Alegre, v. 48, n. 4, p. 256-263, 2017.

GRAYSON, J. Paul. Linguistic capital and academic achievement of Canadian- and foreignborn university students. Canadian Review of Sociology, Toronto, v. 45, n. 2, p. 127-149, 2008. Disponível em: https://www.questia.com/library/journal/1G1-194620370/linguisticcapital-and-academic-achievement-of-canadian. Acesso em: 1 fev. 2018.

HAGELSKAMP, Caroline; SUÁREZ-OROZCO, Carola; HUGHES, Diane. Migrating to opportunities: how family migration motivations shape academic trajectories among newcomer immigrant youth. Journal of Social Issues, Omaha, v. 66, n. 4, p. 717-739, 2010. Disponível em: https://spssi.onlinelibrary.wiley.com/doi/full/10.1111/j.15404560.2010.01672.x. DOI: https://doi.org/10.1111/j.1540-4560.2010.01672.x. Acesso em: 1 fev. 2018.

HAN, Xuesong et al. Report of a mental health survey among chinese international students at Yale University Xuesong. Journal of American College Health, Boston, v. 6, n. 1, 2013. Disponível em: https://www.tandfonline.com/doi/abs/10.1080/07448481.2012.738267. DOI: https://doi.org/10.1080/07448481.2012.738267. Acesso em: 23 abr. 2018.

HSIEN-CHUAN, Patricia Hsu et al. Religion/spirituality and quality of life of international tertiary students in New Zealand: an exploratory study. Mental Health, Religion \& Culture, Coventry, United Kingdom, v. 12, n. 4, p. 385-399, 2009. Disponível em: https://www.tandfonline.com/doi/abs/10.1080/13674670902752920. DOI: https://doi.org/10.1080/13674670902752920. Acesso em: 1 fev. 2018.

HWANG, Bong Joo; BENNETT; Robert; BEAUCHEMIN, James. International students' utilization of counseling services. College Student Journal, Chicago, v. 48, n. 3, p. 347-354, 2014. Disponível em: https://www.questia.com/read/1G1-387058962/international-studentsutilization-of-counseling. Acesso em: 1 fev. 2018.

INSTITUTO NACIONAL DE ESTUDOS E PESQUISAS EDUCACIONAIS - INEP. Censo da educação superior. Brasília, 2017. Disponível em: http://www.andifes.org.br/wpcontent/uploads/2017/04/INEP-Censo-da-Educa\%C3\%A7\%C3\%A3o-Superior-Andifes16042017.pdf. Acesso em: 30 set. 2017.

KAYITESI, Marie Louise; MWABA, Kelvin. South African University Students' Life Satisfaction and Perceptions of African Immigrants. Social Behavior and Personality, Palmerston North, v. 42, n. 7, p. 1127-1132, 2014.

LEE, Ji-Leon; PISTOLE, M. Caroline. International Student Sociocultural Adaptation: Gossip and Place Attachment Contributions. Journal of Mental Health Counseling, Alexandria, VA, v. 36, n. 4, p. 345-359. 2014. Disponível em: https://amhcajournal.org/doi/10.17744/mehc.36.4.0402867150372t21. DOI: https://doi.org/10.17744/mehc.36.4.0402867150372t21. Acesso em: 1 fev. 2018.

LI, Jiaq et al. Attitudes Toward Seeking Professional Counseling Services among Chinese International Students: Acculturation, Ethnic Identity, and English Proficiency. Journal of Multicultural Counseling and Development, Alexandria, VA, v. 44, n. 1, p. 65-76, 2016. 
Disponível em: https://onlinelibrary.wiley.com/doi/full/10.1002/jmcd.12037. DOI: https://doi.org/10.1002/jmcd.12037. Acesso em: 1 fev. 2018.

LIMONTA VIDAL, Elena del Rosario et al. Criterios de los estudiantes extranjeros en torno a su integración al proceso docente-educativo. MEDSAN, Santiago de Cuba, v. 16, n. 8, p. 1250-1255, 2012. Disponível em:

http://scielo.sld.cu/scielo.php?script=sci arttext\&pid=S1029-

30192012000800009\&lng=es\&nrm=iso\&tlng=es. Acesso em: 1 fev. 2018.

LU, Sharon Huixian et al. An internet survey of emotional health, treatment seeking and barriers to accessing mental health treatment among Chinese-speaking international students in Australia. Counselling Psychology Quarterly, Cambridge, v. 27, n. 1, p. 96-108, 2015. Disponível em: https://www.tandfonline.com/doi/abs/10.1080/09515070.2013.824408. DOI: https://doi.org/10.1080/09515070.2013.824408. Acesso em: 1 fev. 2018.

MACIEL, Wellington. Usos de uma cidade da liberdade: estudantes africanos em Redenção. Caderno CRH, Salvador, v. 30, n. 79, p. 189-201, jan./abr. 2010. Disponível em:

http://www.scielo.br/scielo.php?pid=S0103-

49792017000100189\&script=sci_abstract\&tlng=pt. DOI: http://dx.doi.org/10.1590/s010349792017000100012. Acesso em: 1 fev. 2018.

MARGINSON, Simon. La Educación Superior como autoformación: el caso de estudiantes transfronterizos. Revista de La Educación Superior, México, v. 43, n. 169, p. 7-24, 2014.

MARTINS-BORGES, Lucienne. Migração involuntária como fator de risco à saúde mental. Revista Interdisciplinar de mobilidade humana, Brasília, v. 21, p. 151-162, 2013.

MUNGOI, Dulce Maria Domingues Chale João. Ressignificando identidades: um estudo antropológico sobre experiências migratórias dos estudantes africanos no Brasil. Revista

Interdisciplinar Da Mobilidade Humana, Brasília, v. 20, n. 38, p. 125-139, 2012.

Disponível em: http://www.scielo.br/scielo.php?script=sci_arttext\&pid=S1980-

85852012000100008\&lng=pt\&nrm=iso\&tlng=pt. DOI:

https://doi.org/10.1590/S1980-85852012000100008. Acesso em: 1 fev. 2018.

NILSSON, Johanna. E. et al. The Relationships Among Perfectionism, Acculturation, and Stress in Asian International Students. Journal of College Counseling, Alexandria, VA, v. 11, n. 2, p. 147-158, 2008. Disponível em:

https://onlinelibrary.wiley.com/doi/abs/10.1002/j.2161-1882.2008.tb00031.x. DOI:

https://doi.org/10.1002/j.2161-1882.2008.tb00031.x. Acesso em: 1 fev. 2018.

OLIVENCIA, Juan Leiva J. La interculturalidad en el contexto universitario a través de las voces de estudiantes inmigrantes. Revista Electrónica Interuniversitaria de Formación Del Profesorado, Murcia, v. 17, n. 2, p. 155-166, 2014. Disponível em:

http://revistas.um.es/reifop/article/view/197171. DOI:

http://dx.doi.org/10.6018/reifop.17.2.197171. Acesso em: 1 fev. 2018.

ORGANIZAÇÃO INTERNACIONAL PARA AS MIGRAÇÕES - OIM. Glossário sobre Migração. Direito internacional da migração. Genebra: OIM, 2009. n. 22.

PAN, Jia-Yan; WONG; Daniel Fu Keung; YE, Shengquan. Post-migration Growth Scale for 
Chinese International Students: development and validation. Journal of Happiness Studies, Holanda, v. 14, n. 6, p. 1639-1655, 2013. Disponível em: https://link.springer.com/article/10.1007\%2Fs10902-012-9401-z. DOI: https://doi.org/10.1007/s10902-012-9401-z. Acesso em: 1 fev. 2018.

PEI, Penny et al. Stress and quality of life in international and domestic university students: cultural differences in the use of religious coping. Mental Health, Religion \& Culture, Coventry, United Kingdom, v. 15, n. 3, p. 265-277, 2012. Disponível em: https://www.tandfonline.com/doi/abs/10.1080/13674676.2011.571665. DOI: https://doi.org/10.1080/13674676.2011.571665. Acesso em: 1 fev. 2018.

PERROTTA, Daniela. La internacionalización de la universidad: debates globales, acciones regionales. Buenos Aires: EdicionesUNGS, 2015.

PIERONI, Vittorio; FERMINO, Antonia; CALIMAM, Geraldo. Pedagogia da alteridade: para viajar a Cosmópolis. Brasília: Liber Livro, 2014.

PRIETO-WELCH, Susan L. International student mental health. New Directions for Student Services, Ohio, n. 156, p. 53-63, 2016. Disponível em: https://onlinelibrary.wiley.com/doi/full/10.1002/ss.20191. Acesso em: 1 fev. 2018

SALADINO, Caitlin. J.; MARTINEZ, Magdalena. Moving forward: future directions for improving institutional support for latino/a students. New Directions for Higher Education, Bloomington, n. 176, p. 53-67, 2015.

SANTIAGO, Angel Villafañe et al. Necesidades académicas, personales, sociales y vocacionales de estudiantes internacionales y intercambio en la Universidad de Puerto Rico. Revista Electrónica Educare, Heredia, Costa Rica, v. 15, n. 2, p. 185-204, jul./dec. 2011.

SANTOS, Boaventura Souza. Para além do Pensamento Abissal: das linhas globais a uma ecologia de saberes. Novos estudos CEBRAP, São Paulo, n. 79, p. 71-94, 2007.

SHIM, Gayoung et al. Acculturation, self-construal, mental and physical health: An explorative study of East Asian students in Germany. International Journal of Psychology, London, v. 49, n. 4, p. 295-303, 2014. Disponível em: https://onlinelibrary.wiley.com/doi/full/10.1002/ijop.12008. DOI: https://doi.org/10.1002/ijop.12008. Acesso em: 1 fev. 2018.

SILVA, Kelly; MORAIS, Sara Santos. Tendências e tensões de sociabilidade de estudantes dos Palop em duas universidades brasileiras. Pro-Posições, Campinas, v. 23, n. 1(67), p. 163182, jan./abr. 2012.

SOUZA, Luciana Karine de; SEDIYAMA, Cristina Yumi Nogueira. Amizades internacionais: panorama da literatura empírica e um estudo descritivo. Barbarói, Santa Cruz do Sul, n. 36, p. 6-28, jan./jun. 2012.

SUBUHANA, Carlos. A experiência sociocultural de universitários da África Lusófona no Brasil: entremeando histórias. Pro-Posições, Campinas, v. 20, n. 1(58), p. 103-126, jan./abr. 2009. 
SUN, Yi. Chinese students in Japan: the mediator and the moderator between their personality and mental health. International Journal of Psychology, London, v. 48, n. 3, p. 215-223, 2013. Disponível em:

https://onlinelibrary.wiley.com/doi/full/10.1080/00207594.2011.648942. DOI: https://doi.org/10.1080/00207594.2011.648942. Acesso em: 1 fev. 2018.

SWAMI, Viren. Predictors of sociocultural adjustment among sojourning Malaysian students in Britain. International Journal of Psychology, London, v. 44, n. 4, p. 266-273. 2009. Disponível em: https://onlinelibrary.wiley.com/doi/full/10.1080/00207590801888745. DOI: https://doi.org/10.1080/00207590801888745. Acesso em: 1 fev. 2018.

WOODWARD, Judith. Internal state of emergency - working with an international student suffering from trauma in a student counselling service. Psychodynamic Practice, London, v. 16, n. 3, p. 257-271, 2010. Disponível em: https://www.tandfonline.com/doi/abs/10.1080/14753634.2010.493322. DOI: https://doi.org/10.1080/14753634.2010.493322. Acesso em: 1 fev. 2018.

YAKUSHKO, Oksana; DAVIDSON, M. Meghan; SANFORD-MARTENS, Tiffany C. Seeking help in a foreign land: international students' use patterns for a U.S. university counseling center. Journal of College Counseling, Alexandria, VA, v. 11, p. 6-18, 2008. Disponível em: https://onlinelibrary.wiley.com/doi/abs/10.1002/j.2161-1882.2008.tb00020.x. DOI: https://doi.org/10.1002/j.2161-1882.2008.tb00020.x. Acesso em: 1 fev. 2018.

ZAMBERLAM, Jurandir et al. Estudantes internacionais no processo globalizador e na internacionalização do ensino superior. Porto Alegre: Impa Artes Gráficas, 2009. 